\title{
Left main coronary angioplasty performed as a single-day procedure: A modern approach or unnecessary risk?
}

\author{
Andrzej Ciszewski, Paweł Tyczyński, Adam Witkowski \\ Department of Cardiology and Invasive Angiology, National Institute of Cardiology, Warszawa, Poland
}

\author{
Correspondence to: \\ Andrzej Ciszewski, MD, PhD, \\ Department of Cardiology and \\ Invasive Angiology, \\ National Institute of Cardiology, \\ Alpejska 42, 04-628 Warszawa, \\ Poland, \\ phone: +48223434 013, \\ e-mail: aciszewski@ikard.pl \\ Copyright by the Author(s), 2021 \\ Kardiol Pol. 2021; \\ 79 (12): 1393-1394; \\ DOI: $10.33963 /$ KP.a2021.0156 \\ Received: \\ September 9, 2021 \\ Revision accepted: \\ November 5, 2021 \\ Published online: \\ November 5, 2021
}

Several trials proved the feasibility and safety of a single-day coronary angioplasty (SD PCI) in stable angina, however, high-risk patients were underrepresented in the studied groups [1-3]. Therefore, data about SD PCI of the unprotected left main coronary artery (LMCA) bifurcation are sparse and worthy of discussion. The number of SD PCl increases rapidly because it reduces hospital costs and the risk of in-hospital infection; it also increases the number of performed procedures $[2,3]$. At our center, we offer SD PCI to patients without age limits, with stable angina, a possible radial approach, a left ventricular ejection fraction $>30 \%$, normal renal function, domiciled not far from the hospital (transport $<60$ minutes), and no planned $\mathrm{PCl}$ of coronary bypass or chronic total occlusion [4].

Our patient was a 68-year-old man, with type 2 diabetes and hyperlipidemia. He had a history of inferior myocardial infarction treated by angioplasty of the circumflex (Cx) artery with a drug eluting stent (DES) in 2013 and a planned angioplasty with DES of proximal Cx in 2019. He was referred for coronary angiography due to the recurrence of typical angina. The patient had good cardiac contractility and normal renal function. After coronary angiography showing critical ostial stenosis of the left anterior descending (LAD) artery, Medina 0-1-0 (Figure 1A), the patient refused cardiovascular surgery and accepted high-risk angioplasty.

The angioplasty of the unprotected LMCA bifurcation was performed from the radial approach according to the current standards. Figure 1 shows the key steps of the procedure. First, we performed a predilatation of LAD ostial lesion with noncompliant balloons ( 2.5 and $3.5 \times 12 \mathrm{~mm}, 12 \mathrm{~atm}$ ), and, due to dissection at the end of the predilated zone, a DES $(3.5 \times 16 \mathrm{~mm}, 14 \mathrm{~atm})$ was implanted in the LAD around $7 \mathrm{~mm}$ from the ostium. Then a DES $(4.0 \times 18 \mathrm{~mm}, 14 \mathrm{~atm})$ was implanted to the left main and the LAD (Figure 1C). We then used the proximal optimization technique of the LMCA, and intravascular ultrasound (IVUS) to confirm satisfactory lesion dilatation and stent apposition. The next steps were: "kissing" of the LAD/Cx bifurcation (Figure 1C), second proximal optimization technique of the left main (balloon $5.0 \times 8 \mathrm{~mm}, 10 \mathrm{~atm}$ ). A good final angiographic result (Figure 1E) was confirmed by IVUS (Figure 1F). The final IVUS showed correct stent apposition with a minimal stent area of $17 \mathrm{~mm}^{2}$, no dissection, no plaque protrusion.

After the LMCA and LAD angioplasty, the patient remained in a sitting position in an armchair and received a fluid infusion. He was managed according to our protocol [4]. The patient's status was normal, and the electrocardiogram showed no ST-T deviations. The radial artery compression was removed, and the patient left the hospital 8 hours after the $\mathrm{PCl}$. The final IVUS showing a good stent apposition and no dissection was essential in making our decision to discharge the patient on the day of the procedure. The follow-up visit, 3 months and 7 days after the $\mathrm{PCl}$, revealed no complications and no ischemic symptoms. The patient found the early discharge safe and more convenient than the approach with overnight hospitalization.

From this case presentation, we can conclude that the LMCA angioplasty may be safely performed as SD PCI in selected patients at experienced centers and that a confirmation of good angiographic results based on intravascular imaging (IVUS or OCT) after a higher-risk angioplasty is important in early discharge decisions. 


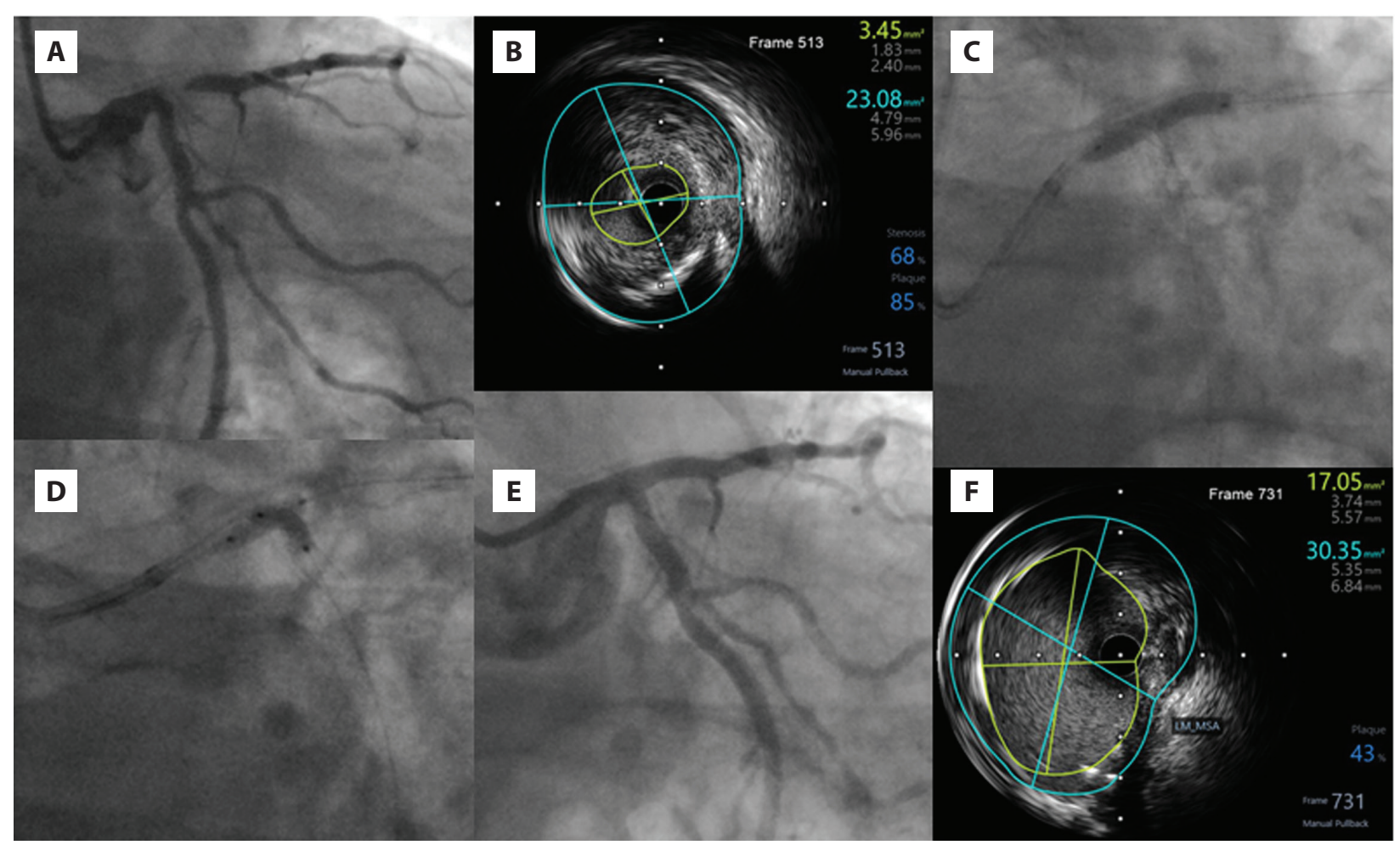

Figure 1. A. Critical ostial LAD stenosis. Severe calcifications in the LM and the proximal LAD. B. IVUS of the LAD ostial lesion. Mix plaque with deep calcifications. Plaque burden over $80 \%$. MLA $3.45 \mathrm{~mm}^{2}$. C. Implantation of DES $(4.0 \times 18 \mathrm{~mm}, 14 \mathrm{~atm})$ to the LM and the LAD. Visible stents in the Cx ostium and the proximal LAD. D. "Kissing" angioplasty of the LAD/Cx bifurcation (NC balloons: $3.0 \times 12 \mathrm{~mm}$ and $3.0 \times 9 \mathrm{~mm}$, $6 \mathrm{~atm})$. E. Final angiographic result. Successful dilatation of the ostial LAD stenosis and stent implantation to the LM and the proximal LAD. No residual stenosis and good stent dilatation in the LM after the final POT with an NC balloon $(5.0 \times 12 \mathrm{~mm}, 10$ atm). F. Final IVUS image showing correct stent apposition with MSA $17 \mathrm{~mm}^{2}$. No dissection on both stent edges, no plaque protrusion

Abbreviations: DES, drug eluting stent; IVUS, intravascular ultrasound; LAD, left anterior descending; LM, left main; MLA, minimal luminal area; MSA, minimal stent area; NC, noncompliant; POT, proximal optimization technique

\section{Article information}

Conflict of interest: None declared.

Open access: This article is available in open access under Creative Common Attribution-Non-Commercial-No Derivatives 4.0 International (CC BY-NC-ND 4.0) license, allowing to download articles and share them with others as long as they credit the authors and the publisher, but without permission to change them in any way or use them commercially. For commercial use, please contact the journal office at kardiologiapolska@ptkardio.pl.

How to cite: Ciszewski A, Tyczyński P, Witkowski A. Left main coronary angioplasty performed as a single-day procedure: A modern approach or unnecessary risk? Kardiol Pol. 2021; 79(12): 1393-1394, doi: 10.33963/KP.a2021.0156.

\section{REFERENCES}

1. Heyde GS, Koch KT, de Winter RJ, et al. Randomized trial comparing same-day discharge with overnight hospital stay after percutaneous coronary intervention: results of the Elective $\mathrm{PCl}$ in Outpatient Study (EPOS). Circulation. 2007; 115(17):2299-2306, doi: 10.1161/CIRCULATIONAHA.105.591495, indexed in Pubmed: 17420341.

2. Taxiarchi P, Kontopantelis E, Martin GP, et al. Same-day discharge after elective percutaneous coronary intervention: insights from the British Cardiovascular Intervention Society. JACC Cardiovasc Interv. 2019; 12(15): 1479-1494, doi: 10.1016/j.jcin.2019.03.030, indexed in Pubmed: 31395218.

3. Amin AP, Pinto D, House JA, et al. Association of same-day discharge after elective percutaneous coronary intervention in the United States with costs and outcomes. JAMA Cardiol. 2018; 3(11): 1041-1049, doi: 10.1001/jamacardio.2018.3029, indexed in Pubmed: 30267035.

4. Ciszewski A. Same-day discharge after coronary angioplasty: data from a single-center registry. Kardiol Pol. 2020; 78(4): 328-330, doi: 10.33963/KP.15196, indexed in Pubmed: 32081841. 